\title{
Psychological Variables in Researches between Social Network and Social Capital
}

\author{
Ran Qu \\ Xi'an Jiaotong University \\ Shaanxi Xueqian Normal University \\ Xi'an, China \\ quran126@stu.xjtu.edu.cn
}

\author{
Xiaoyi Shao \\ Xi'an Jiaotong University \\ Xi'an, China \\ shaoxiaoyi@stu.xjtu.edu.cn
}

\author{
Xiaoli Ni \\ Xi'an Jiaotong University \\ Xi'an, China \\ nixiaoli@mail.xjtu.edu.cn
}

\begin{abstract}
Social network and social capital are critical issues in sociology. There is a rising research tendency on the relationship among psychological variables in social network and social capital. Four common hypotheses were summarized based on comprehensive syntheses, and they were main effect hypothesis, mediator and moderator hypothesis, dynamic effect hypothesis, and synergistic effect hypothesis. Meanwhile, the methodology, definition and assessment should be considered, which induce new thoughts for future studies.
\end{abstract}

Keywords—social network; social capital; psychological variables

\section{SOCIAl Network, Social CAPITAL AND Psychological VARIABLES}

The definition of social capital was formally proposed by the French sociologist Bourdieu in 1980, and then Coleman, Putnam, Portes and his colleagues redefined this concept and conducted a series of researches. Nan Lin [1] thought social capital as resources embedded in social networks which are rooted in social networks and social relations, therefore the social capital can be defined as the resources embedded in a social structure that can be obtained by purposeful actions. Yanjie Bian [2] pointed out that the forms of social capital was the network of relationships in social actors, and the essences was the resources which could be contained in the network and be transformed among social actors. None social actors can unilaterally have such resources, and it must be obtained by the development, accumulation and application of such resources in the network.

At present, in researches on social network and social capital, empirical and quantitative studies gradually become the mainstream, in which the study of that focused on the social status attainment, social support, social stratification and occupational mobility. As continuous expansion of the study of social network and social capital in the social science, the influence were no longer limited to the academic sociology, which became a theme highly researched by scholars from different disciplines, such as psychology. The relationship

This work was supported by the National Social Science Foundation of China under Grant Project: Psychological and Behavioral Model of Adolescent Self-identity Affected by Social Media Networks. No. $15 \mathrm{BSH} 023$ study among social network, social capital and psychological variables show its vitality at its beginning.

Sociology and psychology concern "punctate" (individual) and "linear" (the relationship between individuals) from different perspectives. Sociological researchers were interested in social network and social capital embedded in networks, but psychologists were oppositely concerned about the psychology impacts of social networks on individuals and groups, rather than social network itself. Sociology and psychology to treat persons and their relationships in society is seemingly disparate, but complementary. On the one hand, psychological status of individuals and groups were affected by the characteristics of their social networks; on the other hand, psychological factors of each individual and group had an impact on the formation and development as well as the final characteristics of social networks.

The studies on relationships among social network, social capital and psychological variables were uncommon, but this idea has been had for a long time. In the very beginning, many scholars from social psychology, such as Moreno - the founder of sociometrics, Lewin - the pioneer of group dynamical theory, and Hyde - the proposer of equilibrium theory, believed that social context has an important impact on individual psychology and behavior. In addition, many psychological experiments, such as Hawthorne experiments, conformity experiments also illustrated that social situation had a great effect on human activities [3][4]. So if combined with macro- and medio-scope of sociology and microscopic analysis of psychology, measuring social network and social capital, and meanwhile, taking psychological variables into consideration, the perspectives would be enhanced and the reliability of conclusion would be improved, then we would have a more accurate understanding of human and their society. 


\section{RESEARCH STATUS AND FOUR HYPOTHESES}

Recently, sociology and psychology are both interested in social capital and social network. A psychological factor in social network and social capital focuses on mental health, personality psychology, developmental psychology and management psychology. However, the research of social network, social capital and psychological factors has not yet formed a unified paradigm, and different scholars have different hypotheses. To sum up, there are four common hypotheses, and they are main effects model, mediator and moderator model, dynamic effects model, and synergy effects model. The following four types of model attempt to explain the hypotheses in the study.

\section{A. Maintaining Main Effect Hypothesis}

The main effects model means that social network; social capital and psychological variables have direct effects among themselves, but independent with other variables. According to the direction of impacts, the model can be divided into two categories. One is concerned about the psychological factors effect on social network and social capital; while the other focuses on the influences of social network and social capital with psychological variables.

1) Psychological variables effect on social network and social capital

On the basic view of such studies, the psycho-variables have an impact on social network and social capital, and psychological traits of individuals play its role in maintain and expansion of social network and social capital in the process of achievement.

Mehra, Kilduff and Brass's holistic network study [5] found that the discrepancies of individual self-monitor affect their position and status in social network. Compared with the low self-monitor persons, the higher often occupy the center of social networks. Kalish [6] found a positive correlation among extroversion, density and size of network, but the nervousness is the opposite. In the research of organization creativity, Gloor, Oster, Raz, Pentland and Schoder [7] compared with neuroticism and introversion of the individual, and found that the extroverted person has more probability located in the edge of their social network.

2) Psychological variables effected by social network and social capital

Comparing with the studies above, this type of research is more focused on the interactions between the individual and the social circumstance, trying to find the source of psychological problems in the relationship between individuals and social environment, at the same time; it provides a way for a comprehensive understanding of social network and social capital.

Clifton, Turkheimer and Oltmanns [8][9] had a study on the individual position as an indicator of personality disorder about the social network. Neal found curve effect on the centrality of children's social network of their aggressive behaviors. The organization creativity studied by Gloor [7] found a positive correlation between high creativity of employees and changes of their centrality. Then, Zhou, Shin, Brass and Choi [10] found a curvilinear relationship between the creativity of employees and their weak ties. The individual network analyzed by Falci and McNeely [11] showed a curved linear relation between the size of friend-network and depressive symptoms of teenagers.

Similarly, scholars also have a series of studies about that in China. Winnie Yip et al.[12] found a positive correlation between social capital and mental health in rural China. Yuxia Li had an empirical study about the relation between rural social capital and mental health, and found the frequency they meet friends, reciprocity and mutual-benefits were the factors affected mental health. Hui $\mathrm{Yu}$ analyzed the data from a sampling survey about Shanghai residents, and the outcome showed that individuals and collective social capital had effects on mental health. Yandong Zhao had a discussion on mental and physical health impact of social network between urban and rural residents. Some scholars further studied on theoretical analysis of relationship between social capital and mental health [13].

In summary, the main effects model is a primary pattern in the study of social networks, social capital and psychological factors. There are direct effects on the relationship among social networks, social capital and psychological variables, and it has achieved abundant researches, and lay a foundation for further study. However, in some situations, this hypothetical model appears to be inadequate in a full explanation on the complex relationship among social networks, social capital and psychological factors.

\section{B. Mediator and Moderator Hypothesis}

Mediator and moderator model infers that there are some other variables impact on the relationship among social network, social capital and psychological variables. Generally speaking, it pays attention to 3 sets of variables: 1) social network and social capital; 2) psychological variables (factors) 3 ) others, among which are relationships of mediator and moderator effects. In the mediator assumption, independent variables have impacts on the dependent variable through mediator variables; in the moderator assumption, the moderator variables affect the power of independent variables on dependent variables. Compared with main effect hypothesis this one gives a more nuanced explanation to the relationship among social networks, social capital and psychological factors that is closer to the reality in some way. For example, it is more convinced to describe that the individual social capital affect mental health through pressure - buffer mechanism, rather than directly explain that social capital has effects on mental health.

Nan Lin has an earlier contribution in this field. $\mathrm{He}$ believes that human health, mainly physical health is affected by two environmental factors - stress and resources, and social resources play a buffer role on social and psychological pressure when they affect individual health. Liu and Ipe [14] examined out the mediator effect on the interpersonal citizenship behavior (ICB) between personality (responsibility and agreeableness) and network centrality of group members in their studies, in other words, the more responsibility and agreeableness the employees have, the more interpersonal citizenship behavior would be demonstrated easily, therefore, the person who has those traits is more likely to be the central 
in the team. Chinese scholars have found that social networks between psychological empowerment of migrant workers and organizational citizenship behavior have a moderator role [15].

\section{Dynamic Effect Hypothesis}

Dynamic effects model hypothesizes that social networks, social capital and psychological factors construct and influence among each other, and individuals construct their networks actively (e.g. selection effect), while social networks and social capital, in turn, affect individual's psychology and behavior (e.g. influence effect). The influence is not as the main effects model as a one-way impact, but in mutual. Individual shapes and also is shaped by social network.

In mental health research, studies have shown that the lower social capital is, the worse mental health, while the worse mental health is, result in decline or difficult to increase social capital, and create a vicious cycle. In the studies of developmental psychology, Mercken et al [16]. used a vertical method of social network analysis to test for smoking behavior based on gender differences, and have a result that it is no differences in choosing a friend who is smoking among different gender of teenagers, however, female adolescents are more susceptible to the friends who are smoking. Mercken et al [17]. Through longitudinal studies on relation between smoking behavior and dynamic network of youngster, found the selection effect and the impact effect are both playing an important role in the process of smoking behavior development of adolescents, and the selection effect was greater than the impact effect.

\section{Synergistic Effect Hypothesis}

Synergy effects model assumes that social networks, social capital and psychological variables can be used to explain the state of the individual and social phenomena, and they are coefficient. The study of this model is not much, and human, social and psychological capital of human resources can be seen as an attempt of this model. Human and social capital is playing an increasingly important role in the performance and the competitiveness of organizations, which become a consensus in theory and practice. But in recent years, positive organizational behavior suggested that there is a need to shift to a new paradigm for the effective management on organizations that is exploring psychological capital in order to create sustainable competitive advantages. In this theory, social capital and psychological capital is coefficient to the performance of individuals and organizations.

With the present research, the coexistence of different modes in a variety of studies is becoming prevalent. The four distinctive hypotheses above describe the relationship of social network, social capital and psychological variables from different perspectives, which is progressive and complementary each other. The results of four modes are not same, nor achieve the integration between modes, and social networks, social capital and mental mechanism between variables remains to be further studied. However, it has different outcomes under the studies of four models above, nor achieves integration among them. It means that the mechanism of social networks, social capital and psychological variables remains to be further studied.

\section{INTROSPECTION AND PROSPECTION}

The study of psychological variables in social networks and social capital is promising, and it provides researchers and scholars a broad room to reconsider and apply this method in theory and practice. The following is introspection for the existed studies from research hypotheses, study levels, and subjects, etc., and prospection for the discussion on future efforts and further research

\section{A. Analysis from Research Hypotheses}

From the models of research hypotheses in the current studies, main effects model are used much more than that of mediator and moderator model, dynamic effects model, and synergy effects model in the research of social networks, social capital and psychological factors. Although it is benefit to explore the relationship among social networks, social capital and psychological factors respectively, it underestimates the complexity of relationship among them, and impede further exploration and study.

\section{B. Analysis from Study Levels}

Current researches have mainly focused on medium- and micro-scaled empirical studies, while macro-level public policy studies and theoretical exploration are relatively weak [18]. It is difficult to establish the theoretical contact with the traditional disciplines for lack of theoretical foundations, and it is not conducive to sustainable development in research, because lack of a unified framework hinders effective and reliable theories in the study of hypotheses. There are still few effect on policies and public services, which lead to an embarrassing situation in little theoretical foundation and practical application.

\section{Analysis from Research Fields}

Currently, it is relatively narrow for the studies of psychological variables, and most of them concentrate on personality psychology, management psychology, developmental psychology and mental health. The limitations of disciplines restrict the diversity of theoretical assumptions and research methods. It could be considered to further expanding research in many fields, such as expansion of the quantitative studies and species of psychological variables, and extend studies into many other areas in educational psychology, consumer psychology, and emotional psychology, etc.

\section{Analysis from Definition and Measurement}

There are a lot of divergence in the definition of social capital, social networks and psychological variables, but the measurement is more casual. There are also some difficulties in comparison of results among each other, and it is not conducive to the accumulation and development of studies. In general, it is meticulous of social networks and social capital measurement in sociology, while it is not standardized in the measurement of psychological variables; however, psychologists carefully measure the psychological variables, on the contrary, social networks and social capital measurement is rather rough and casual. It must be imminent for a multidisciplinary integration. In addition, measurement instruments have rarely reliability and validity analysis, which have not been tested. 


\section{E. Analysis from Methodologies}

Firstly, many studies in this field are belonging to quantitative research, but lack of qualitative research lead to difficulties in accurately revealing the causation and mechanism behind the statistical results. Secondly, most present researches are cross-sectional study and few longitudinal studies, so it is difficult to distinguish the logic between social capital and psychological variables. Therefore, it is important for realization that causal relationship between social capital and psychological factors in longitudinal methods. A series of comprehensive and innovative research methods in the field should be ready.

\section{F. Zeitgeist and Localization}

The related studies and results in China are much less. In future studies, there is one way could be put forward that except to solve the issues above, and have a concern on the present ages, studies should be combined with national actual situation. Further researches can be set out to exploring the relationship between social capital and psychological variables on the Internet; the relation of social capital and employee psychological in the social organizations; the research about social capital and psychological variables of vulnerable groups in the process of China's social transformation; the study on the elder, the rural, the western, and other special groups in China, and so on.

\section{CONCLUSION}

In all, the study of social networks, social capital and psychological variables is great promising, and it is not only need a balanced development with comprehensive studies on hypotheses and methodologies, but also multi-disciplinary works should be sought from the theories of traditional disciplines to expand research and feedback practical requirements. Then, the relationship among social network, social capital and psychological variables will be much clearer, and the studies can be full of vigor and vitality to have a further contribution with proper traits and values.

\section{REFERENCES}

[1] Nan Lin, and Walter M. Ensel. (1989). Life stress and health: stressors and resources. American Sociological Review. 54(3):382-399
[2] Yanjie Bian (2004). The sources and effects of urban social capital: the perspectives and findings of networks. China Social Science, 3, 136-141. (In Chinese)

[3] Pattison, P., \& Robins, G. (2004). Building models for social space: neighborhood-based models for social networks and affiliation structures. Mathematics and Social Sciences,4, 11-29.

[4] Robins, G., \& Kashima, Y. (2008). Social psychology and social networks: Individuals and social systems. Asian Journal of Social Psychology, 11, 1-12.

[5] Mehra, A., Kilduff, M., \& Brass, D. J. (2001). The social networks of high and low self-monitors: implications for workplace performance. Administrative Science Quarterly, 46(1), 121-146.

[6] Kalish, Y., \& Robins, G. (2006). Psychological predispositions and network structure The relationship between individual predispositions, structural holes and network closure. Social Networks, 28, 56-84.

[7] Gloor, P., Oster, D., Raz, O., Pentland, A., \& Schoder, D. (2010). The virtual mirror reflecting on your social and psychological self to increase organizational creativity. Journal on International Studies of Management \& Organization, 40(2).

[8] Clifton, A., Turkheimer, E., \& Oltmanns, T. F. (2007). Improving assessment of personality disorder traits through social network analysis. Journal of Personality, 75(5), 1007-1031.

[9] Clifton, A, Turkheimer, E, \& Oltmanns, T. F. (2009). Personality disorder in social networks: network position as a marker of interpersonal dysfunction. Social Networks, 31, 26-32.

[10] Zhou, J., Shin, S. J., Brass, D. J. \& Choi, J. (2009). Social networks, personal values, and creativity: Evidence for curvilinear and interaction effects. Journal of Applied Psychology, 94(6), 1544-1552.

[11] Falci, C., \& McNeely, C. (2009). Too many friends: socialintegration, network cohesion and adolescent depressive symptoms. Social Forces, 87(4), 2031-2062.

[12] Winnie Yip, S. V. Subramanian, Andrew D. Mitchell, Dominic T. S. Lee Jian Wang, Ichiro Kawachi,. (2007). Does social capital enhance health and well-being: evidence from rural China. Social Science \& Medicine 64: 35-49

[13] Richard M. Scheffler, Timothy T. Brown. (2008). Social capital, economics and health: new evidence. Health, Economics, Policy and Law 3:321-331

[14] Liu, Y., \& Ipe, M. (2010). How do they become nodes? Revisiting team member network centrality. The Journal of Psychology, 144(3), 243-258.

[15] Yu, L., Yike, R., Juan, X. (2012). Migrant workers empowerment and organizational citizenship behavior - the moderator effect of social networks. Management modernization, 4, 91-93. (In Chinese)

[16] Mercken, L., Snijders, T. A. B., Steglich, C., Vertiainen, E., \& Vries, H. D. (2010a). Smoking-based selection and influence in gender-segregated friendship networks: a social network analysis of adolescent smoking. Addiction, 105(7), 1280-1289.

[17] Mercken, L., Snijders, T. A. B., Steglich, C., Vartiainen, E.,\& Vries, H. D. (2010b). Dynamics of adolescent friendship networks and smoking behavior. Social Networks, 32, 72-81.

[18] Yaofeng Li (2012). A critical review of the relation between social capital and mental health. Future and development, 4, 18-22. (In Chinese) 